\title{
Enfermedad ósea metabólica renal en el potencial receptor de trasplante renal
}

\author{
Renal metabolic bone disease in the potential \\ kidney transplant recipient \\ Juan Carlos Ramírez-Sandoval* \\ * Departamento de Nefrología y Metabolismo Mineral. Instituto Nacional de Ciencias Médicas y Nutrición «Salvador Zubirán».
}

\section{TRASTORNOS MINERALES Y ÓSEOS \\ EN LA ENFERMEDAD RENAL CRÓNICA (TMO-ERC) EN EL POTENCIAL RECEPTOR}

\section{Resumen}

\section{Respecto al diagnóstico previo al trasplante de TMO-ERC}

1. Los receptores candidatos a trasplante renal deberán tener al menos una medición de las concentraciones séricas de calcio, fósforo, albúmina y fosfatasa alcalina dentro del mes previo al trasplante y una medición de las concentraciones de hormona

\begin{tabular}{ll}
\hline & \multicolumn{1}{c}{ Nomenclatura y descripción } \\
\multicolumn{1}{c}{ Término } & \multicolumn{1}{c}{ Definición } \\
\hline Obligatorio & Se debe de incorporar en todos los casos. \\
Sugerido & Se invita a ser incorporado en la mayoría de los casos. \\
Deseable & Se considera necesario, pero no es una prioridad. \\
\hline
\end{tabular}

Abreviaturas:

$\mathrm{ERC}=$ Enfermedad renal crónica.

$\mathrm{ERCT}=$ Enfermedad renal crónica terminal.

IPTH = Hormona paratiroidea molécula intacta.

iFGF23 = Factor de crecimiento derivado de fibroblastos 23 molécula intacta.

TMO-ERC $=$ Trastornos mineral y óseos de la enfermedad renal crónica. paratiroidea molécula intacta (iPTH) dentro de los 3-6 meses previo al trasplante (Obligatorio).

2. La presencia hipercalcemia con hiperparatiroidismo en receptores requiere diagnóstico y tratamiento previo a la cirugía de trasplante renal por sus implicaciones pronósticas. Por tal razón, se sugiere la determinación de calcio libre (ionizado) dada la alta frecuencia de errores diagnósticos en la interpretación del calcio utilizando el calcio total o corregido para albúmina (Sugerencia).

3 . Se sugiere no medir previo al trasplante rutinariamente isoformas de vitamina $D$ (25-hidroxi vitamina D y 1,25-dihidroxivitamina D), marcadores específicos de recambio óseo basados en la síntesis de colágeno u otros tales como iFGF23, esclerostina o Klotho circulante (Sugerencia).

4. Es deseable la realización de densitometría ósea tres meses después del trasplante renal, en pacientes con función renal estable, en tratamiento con esteroides y con factores de riesgo para osteoporosis. Opcionalmente, el estudio puede realizarse previamente a la cirugía de trasplante, pero no es indispensable como preparación al trasplante (Deseable).

5. Es deseable la realización de biopsia ósea únicamente en aquellos potenciales receptores en los cuales el conocimiento del tipo de osteodistrofia renal modificaría alguna decisión terapéutica (Deseable). 
Respecto a las consideraciones de tratamiento de los TMO-ERC previo al trasplante renal

6. Previo al trasplante se deberán corregir la presencia de las alteraciones bioquímicas. Sin embargo, la realización del trasplante renal es la medida de tratamiento más eficaz para los TMO-ERC y en la mayoría de los casos los trastornos del TMO-ERC no debieran ser motivo de exclusión permanente o dilación del procedimiento del trasplante renal (Sugerencia).

7. No se conocen los límites en las concentraciones de iPTH óptimos en los pacientes en diálisis en preparación para un trasplante, por lo cual se recomienda no suspender o posponer el trasplante renal basado únicamente en cifras de iPTH, especialmente en pacientes asintomáticos, con síntomas leves y con normocalcemia (Sugerencia).

8. Se sugiere la realización de paratiroidectomía parcial previa al trasplante en los siguientes casos: (I) hiperparatiroidismo secundario grave refractario a tratamiento médico, generalmente con cifras de PTH mayor a $1,000 \mathrm{pg} / \mathrm{mL}$, con síntomas secundarios (por ejemplo con fracturas, dolor óseo, anemia secundaria) y cuyo tiempo de espera del trasplante sea mayor de 6-12 meses, (II) aquellos casos con hiperparatiroidismo e hipercalcemia sostenida, no secundaria a fármacos, y (III) presencia de arteriolopatía cálcico urémica (calcifilaxis) asociadas con cifras de PTH mayores de 1,000 pg/mL (Sugerencia).

9. La presencia de calcificaciones vasculares o valvulares aumenta el riesgo cardiovascular en los potenciales receptores, pero el proceso de calcificación se atenúa o se resuelve con el trasplante renal y no debe ser una contraindicación para el procedimiento quirúrgico (Sugerencia).

\section{Respecto a las consideraciones en el donador renal vivo}

10. Se deberá realizar la valoración sobre el riesgo de fracturas y la suplementación de vitamina $D$ de acuerdo con las recomendaciones de población general en el donador renal (Sugerencia).

\section{Respecto al diagnóstico previo al trasplante de TMO-ERC}

\section{Sustento científico}

1. Los receptores candidatos de trasplante renal deberán tener al menos una medición de las concentraciones séricas de calcio, fósforo, albúmina y fos- fatasa alcalina dentro del mes previo al trasplante y una medición de las concentraciones de hormona paratiroidea molécula intacta (iPTH) dentro de los 3-6 meses previo al trasplante (Obligatorio).

De acuerdo con las recomendaciones internacionales y regionales, ${ }^{1}$ la atención de todos los pacientes con ERCT incluye el diagnóstico y tratamiento oportuno de las alteraciones bioquímicas de los TMO-ERC. En México, se reconoce la dificultad de la medición frecuente de la iPTH dada la variabilidad de los ensayos disponibles, la accesibilidad para una medición frecuente y/o limitaciones de índole económica. Sin embargo, es recomendable que aquellos centros dedicados al trasplante renal tengan la capacidad para realizar el diagnóstico oportuno y el tratamiento eficaz de los TMO-ERC antes y después del trasplante renal, incluyendo la atención del hiperparatiroidismo. Un centro de atención que realiza trasplante renal deberá contar con la infraestructura para realizar el diagnóstico de las anormalidades bioquímicas de acuerdo con las guías de tratamiento nacionales e internacionales. De acuerdo con las guías de la Sociedad Latinoamericana de Nefrología e Hipertensión (SLANH), en pacientes con ERC estadio 5 y $5 \mathrm{D}$, la medición del calcio y fósforo debe realizarse cada uno a tres meses, la de iPTH y fosfatasa alcalina cada tres a seis meses. ${ }^{2}$ Por tal motivo, se ha considerado pertinente que los centros de trasplante renal cuenten con una medición de todos estos parámetros, incluyendo la iPTH, por lo menos en los seis meses previos al trasplante.

2. La presencia de hipercalcemia con hiperparatiroidismo en receptores requiere diagnóstico y tratamiento médico o quirúrgico previo a la cirugía de trasplante renal. Por tal razón, se sugiere la determinación de calcio libre (ionizado) dada la alta frecuencia de errores diagnósticos en la interpretación del calcio utilizando el calcio total o corregido para albúmina (Sugerencia).

El estándar de referencia para la determinación de las concentraciones de calcio es la medición de calcio ionizado. La medición de calcio ionizado es complicada dado el manejo y resguardo de las muestras, razón por la cual habitualmente se utilizan las concentraciones de calcio total o calcio corregido para las cifras de albúmina. Desafortunadamente, las mediciones del calcio total o corregido para la albúmina son imprecisas y conllevan el riesgo de una gran cantidad de diagnósticos erróneos. ${ }^{3,4} \mathrm{La}$ 
presencia de hiperfosfatemia, alteraciones del $\mathrm{pH}$ e hipoalbuminemia en pacientes con ERCT son factores asociados al diagnóstico de falsa hipercalcemia o falsa normocalcemia (hipo o hipercalcemia oculta), razón por la cual se ha recomendado la medición de calcio ionizado. ${ }^{5}$ La presencia de hipercalcemia oculta tiene una fuerte asociación con mortalidad en sujetos en diálisis. ${ }^{3}$ La presencia de hipercalcemia persistente, no asociada al uso de medicamentos, es una de las indicaciones mejor reconocidas de paratiroidectomía previa al trasplante, por lo cual los autores de estas guías recomiendan la medición del calcio ionizado en potenciales receptores de trasplante.

3. Se sugiere no medir previo al trasplante rutinariamente isoformas de vitamina $D$ (25 hidroxivitamina D y 1,25 dihidroxivitamina D), marcadores específicos de recambio óseo basados en la síntesis de colágeno u otros tales como iFGF23, esclerostina o Klotho circulante (Sugerencia).

Existe poca información sobre la utilidad de estas mediciones en el estudio del potencial receptor renal. Esta recomendación es consistente con aquéllas publicadas en guías internacionales. ${ }^{1}$

4. Es recomendable la realización de densitometría ósea tres meses después del trasplante renal, en pacientes con función renal estable, en tratamiento con esteroides y con factores de riesgo para osteoporosis. Opcionalmente, el estudio puede realizarse previamente a la cirugía de trasplante, pero no es indispensable como preparación al trasplante (Deseable).

Evidencia reciente ha mostrado que la densidad ósea baja detectada por densitometría ósea en pacientes con ERC G5 predice el riesgo de fracturas. ${ }^{6-8}$ Sin embargo, las opciones de tratamiento para prevenir fracturas en sujetos en diálisis generalmente se limitan al control de las anormalidades bioquímicas. El empleo de otros medicamentos utilizados en osteoporosis, tales como antirresortivos, es controversial. Después del trasplante renal, el riesgo de fracturas es alto al agregarse a la enfermedad ósea preexistente durante el periodo de uremia, los factores propios del trasplante. ${ }^{9}$ Se ha demostrado que existe una rápida pérdida de la masa ósea en el periodo postrasplante temprano, habitualmente en la zona trabecular ósea, atribuida tradicionalmente al uso de esteroides. ${ }^{10}$ Sin embargo, aún con los esquemas inmunosupresores libres de esteroides, existe un alto riesgo de pérdida de masa ósea postrasplante en regiones óseas "no centrales", tales como el hueso del radio." Un estudio retrospectivo en 238 pacientes receptores de trasplante renal demostró que la densidad ósea baja estaba asociada con un mayor riesgo de fracturas. ${ }^{12}$ Por tales motivos, se ha sugerido que la densitometría se realice por lo menos tres meses después del trasplante renal en receptores con función del injerto estable (tasa de filtrado glomerular $>30 \mathrm{~mL} /$ $\min / 1.73 \mathrm{~m}^{2}$ ), que han recibido esteroides y que tienen los factores de riesgo para osteoporosis indicados en la Tabla 1,9 en concordancia con la última actualización de las guías internacionales KDIGO 2017. Las definiciones de osteoporosis en sujetos con trasplante renal son similares a las propuestas en población general, basados en criterios cuantitativos del resultado de la absorciometría dual por rayos $\mathrm{X}$ en los cuales un puntaje $T \leq-2.5$ es diagnóstico de osteoporosis. En estos sujetos con densidad ósea mineral baja, el empleo de vitamina $D$ y/o antirresortivos óseos durante los 12 meses posteriores al trasplante renal está justificado. ${ }^{1}$ No existe evidencia sobre la frecuencia ideal para repetir la densitometría ósea en sujetos con trasplante renal después del primer año.

5. Es deseable la realización de biopsia ósea únicamente en aquellos potenciales receptores en los cuales el conocimiento del tipo de osteodistrofia renal modificaría alguna decisión terapéutica (Deseable).

La biopsia de hueso permite evaluar la calidad ósea y el tipo de alteración según el recambio óseo, la mineralización y el volumen. ${ }^{1}$ Después del trasplante renal existe una disminución del recambio óseo observado en estudios de histomorfometría. En una cohorte contemporánea de sujetos con trasplante renal sometidos a biopsia pre y posterior a la cirugía y con esquemas de inmunosupresión actuales, se encontró que la proporción de pacientes con alto recambio óseo disminuyó de 63 a 19\% después del trasplante en comparación con la proporción de pacientes con bajo recambio óseo, cuya frecuencia aumentó de 26 a 52\%. Desafortunadamente, los valores de $\mathrm{T}$ medidos por densitometría y los biomarcadores de recambio óseo no tuvieron asociación con los hallazgos por histomorfometría en esta cohorte. ${ }^{13}$ Los clínicos 
Tabla 1: Factores de riesgo asociados a la pérdida de masa ósea y fracturas.

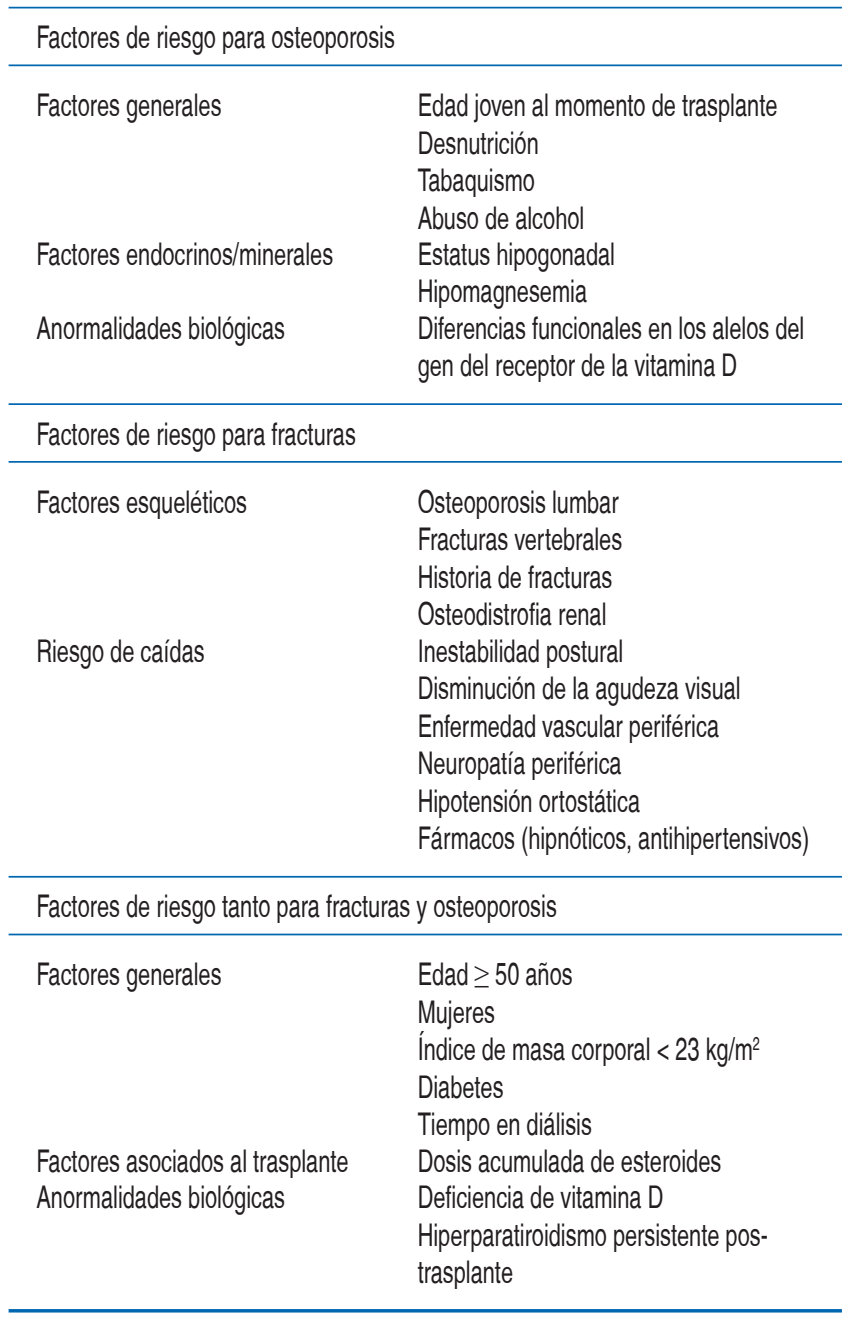

Adaptado de: Bouquegneau A, Salam S, Delanaye P, Eastell R, Khwaja A. Bone Disease after Kidney Transplantation. Clin J Am Soc Nephrol. 2016; 11 (7): 1282-1296.

deben tener presente que los métodos no invasivos tales como la densitometría o los biomarcadores séricos óseos tienen una asociación limitada con los hallazgos de la biopsia ósea. Actualmente existen pocos centros hospitalarios en México con la capacidad para realizar estudios histomorfométricos de biopsias óseas.

Respecto a las consideraciones de tratamiento de los TMO-ERC previo al trasplante renal

6. Previo al trasplante se deberán corregir la presencia de las alteraciones bioquímicas. Sin embargo, la realización del trasplante renal es la medida de tratamiento más eficaz para los TMO-ERC y en la mayoría de los casos los trastornos del TMO-ERC no debiera ser motivo de exclusión permanente o dilación del procedimiento del trasplante renal (Sugerencia).

En la mayoría de los receptores, el trasplante renal mejora o soluciona el hiperparatiroidismo secundario al disminuir las concentraciones de iPTH después del trasplante independientemente de las concentraciones iniciales. ${ }^{14-16} \mathrm{EI}$ trasplante renal normaliza las cifras de iPTH en 55 a $80 \%$ en un periodo de uno a dos años. ${ }^{17}$

Entre 25 a $50 \%$ de los receptores de trasplante renal persisten con concentraciones elevadas de iPTH al largo plazo debido a la transformación adenomatosa de los nódulos paratiroideos, una absorción intestinal deficiente del calcio secundaria al uso de esteroides y a una frecuente suplementación inapropiada de vitamina $D$ nutricional. ${ }^{18,19}$ Existe el riesgo potencial de que aquellos sujetos sin una resolución adecuada del hiperparatiroidismo postrasplante presenten desenlaces adversos renales ${ }^{17}$ y la realización de la paratiroidectomía posterior al trasplante pudiera implicar un riesgo de deterioro en la función del injerto. ${ }^{20}$ Algunos estudios observacionales sugieren que la realización de paratiroidectomía previa al trasplante pudiera mejorar el riesgo a largo plazo del injerto renal. ${ }^{21}$ Sin embargo, la realización de paratiroidectomía en hiperparatiroidismo severo previo al trasplante conlleva un alto riesgo de complicaciones tales como hipocalcemia sostenida, ${ }^{22}$ riesgo de hospitalizaciones ${ }^{23} \mathrm{y}$ un mayor tiempo de espera en diálisis, lo cual aumenta el riesgo de complicaciones y muerte. Además, el riesgo de hipoparatiroidismo o concentraciones muy bajas de iPTH pretrasplante está asociado con riesgos tales como una pobre función del injerto, eventos cardiovasculares o muerte. ${ }^{24}$ Por tales motivos, se sugiere que en los sujetos con hiperparatiroidismo, especialmente aquéllos con concentraciones de IPTH mayores de nueve veces el límite superior de referencia del reactivo utilizado, exista un seguimiento estrecho posterior al trasplante con mediciones seriadas de iPTH, calcio, fósforo y fosfatasa alcalina total. En aquellos casos con hiperparatiroidismo e hipercalcemia persistente después del trasplante y repercusión clínica (fracturas, baja densidad mineral ósea, hipercalciuria, litiasis) se deberá 
valorar la realización de paratiroidectomía o el uso de calcimiméticos, tomando en cuenta que un grupo de pacientes puede normalizar las alteraciones bioquímicas en un periodo de hasta dos años después del trasplante. ${ }^{25,26}$ No existe evidencia sobre la mejor estrategia de tratamiento en estos casos.

7. No se conocen los límites en las concentraciones de iPTH óptimos en los pacientes en diálisis en preparación para un trasplante, por lo cual se recomienda no suspender o posponer el trasplante renal basado únicamente en cifras de iPTH, especialmente en pacientes asintomáticos, con síntomas leves y con normocalcemia (Sugerencia).

No existe un consenso actual sobre los límites aceptables en las concentraciones de la iPTH de sujetos en diálisis candidatos a un trasplante renal. De acuerdo con recomendaciones de la iniciativa para desenlaces renales América (The National Kidney Foundation Kidney Disease Outcome, KDOQI), una cifra de iPTH $>800$ $\mathrm{pg} / \mathrm{mL}$ definía hiperparatiroidismo severo. ${ }^{27} \mathrm{El}$ límite de referencia de $800 \mathrm{pg} / \mathrm{mL}$ históricamente se consideraba como una indicación habitual para la realización de una paratiroidectomía en personas en diálisis. Recientemente, la KDIGO (Kidney Disease Improving Global Outcome) define concentraciones altas de iPTH según la tendencia individual de cada paciente o, si no se dispone de varias mediciones históricas, utilizar un corte de nueve veces el límite superior de referencia del ensayo (por ejemplo, 585 pg/ $\mathrm{mL}$ si el límite superior del ensayo utilizado es mayor a $65 \mathrm{pg} / \mathrm{mL}$ ). ${ }^{28}$ Sin embargo, son escasos los estudios prospectivos que demuestren una cifra de iPTH en la cual se debiera posponer la cirugía de trasplante. Sujetos con cifras de iPTH mayores de $1,000 \mathrm{pg} / \mathrm{mL}$, sin hipercalcemia, pueden mostrar resolución del hiperparatiroidismo después de la cirugía. El grupo de autores del consenso sugiere que el tratamiento del hiperparatiroidismo sea individualizado de acuerdo con los riesgos del potencial receptor.

8. Se sugiere la realización de paratiroidectomía parcial previa al trasplante en los siguientes casos: (I) hiperparatiroidismo secundario grave refractario a tratamiento médico, generalmente con cifras de PTH mayor a 1,000 pg/mL, con síntomas secundarios (por ejemplo con fracturas, dolor óseo, anemia secundaria) y cuyo tiempo de espera del trasplante sea mayor a 6-12 meses, (II) aquellos casos con hiperparatiroidismo e hipercalcemia sostenida, no secundaria a fármacos, e hiperfosfatemia refractaria, (III) presencia de arteriolopatía cálcico urémica (calcifilaxis) asociadas a cifras de PTH mayores de $1,000 \mathrm{pg} / \mathrm{mL}$ (Sugerencia).

No existe una definición universal de hiperparatiroidismo refractario, pero generalmente se define como un hiperparatiroidismo sin respuesta a tratamiento médico con una adecuada dosis de diálisis, quelantes de fósforo y calcimiméticos. Metaanálisis recientes sugieren que la paratiroidectomía muestra un importante efecto de mejoría en mortalidad, riesgo de fracturas, riesgo de hospitalizaciones y calidad de vida en sujetos con hiperparatiroidismo refractario con ERCT. ${ }^{22,29}$ La hipercalcemia con hiperfosfatemia refractaria es la principal indicación de paratiroidectomía, pues la cirugía reduce efectivamente las concentraciones de calcio y fósforo. ${ }^{30-32}$ Cinacalcet, un modulador alostérico de calcio, es un tratamiento que disminuye las concentraciones de iPTH y normaliza las concentraciones de calcio en casos de hiperparatiroidismo terciario. En una búsqueda sistemática reciente con 47 estudios, se encontró que la tasa de curación con paratiroidectomía total o subtotal previo al trasplante fue de $96 \%$. En comparación, el tratamiento con cinacalcet pre y posterior al trasplante, normalizó el calcio sérico en $81 \%$ de los trasplantados. Sin embargo, se observó gran variabilidad de la corrección de la hipercalcemia entre los estudios con cinacalcet, con tasas de respuesta entre 28 a 100\%; además que la dosis de cinacalcet utilizada fue variable (30 a $180 \mathrm{mg} / \mathrm{día}$ ) y su suspensión en el periodo temprano postrasplante se asoció con una recaída en la hipercalcemia. ${ }^{33}$ Considerando el costo efectividad de la paratiroidectomía y cinacalcet, se consideró la paratiroidectomía parcial como primera opción en aquellos casos con hipercalcemia sostenida (hiperparatiroidismo terciario).

La presencia de calcifilaxis asociada con hiperparatiroidismo es una indicación de paratiroidectomía. ${ }^{34} \mathrm{Si}$ bien es una manifestación poco frecuente, su presencia es motivo de trasplante anticipado en varios centros dada su resolución o mejoría después del trasplante. ${ }^{35,36}$

9. La presencia de calcificaciones vasculares o valvulares aumenta el riesgo cardiovascular en los potenciales receptores, pero el proceso de calci- 
ficación se atenúa o se resuelve con el trasplante renal y no debe ser una contraindicación para el procedimiento quirúrgico (Sugerencia).

Si bien la presencia de calcificaciones vasculares aumenta el riesgo de muerte y de muerte cardiovascular, ${ }^{37}$ no existe información suficiente para descartar el trasplante renal en pacientes con diversos grados de calcificación. Existe poca información sobre el efecto del trasplante en el proceso de la calcificación vascular.

\section{Respecto a las consideraciones en el donador vivo de trasplante renal}

10. Se deberá realizar la valoración sobre el riesgo de fracturas y la suplementación de vitamina $D$ de acuerdo con las recomendaciones de población general en el donador renal (Sugerencia).

Existe poca información sobre los TMO-ERC en donadores renales sanos. Se recomienda utilizar las recomendaciones generales propuestas por las guías nacionales para población general, en los cuales se deben investigar los factores de riesgo para osteoporosis. Entre estos destacan: postmenopausia, menopausia temprana (antes de los 45 años), edad mayor a 65 años, antecedente familiar de fractura de cadera antes de los 75 años, historia de fractura vertebral por compresión, índice de masa corporal menor a $19 \mathrm{~kg} /$ $\mathrm{m}^{2}$ y otras patologías que son poco frecuentes en sujetos candidatos a donación renal (artritis reumatoide, hiperparatiroidismo primario, hipogonadismo, síndromes de mala absorción intestinal, diabetes mellitus 2).

\section{REFERENCIAS}

1. Kidney Disease: Improving Global Outcomes (KDIGO) CKDMBD Update Work Group. KDIGO 2017 Clinical Practice Guideline Update for the Diagnosis, Evaluation, Prevention, and Treatment of Chronic Kidney Disease-Mineral and Bone Disorder (CKD-MBD). Kidney Int Suppl (2011). 2017; 7 (1): 1-59.

2. Bellorin-Font E, Ambrosoni P, Carlini RG, Carvalho AB, CorreaRotter R, Cueto-Manzano A et al. Guías de práctica clínica para la prevención, diagnóstico, evaluación y tratamiento de los trastornos minerales y óseos en la enfermedad renal crónica (TMO-ERC) en adultos. Nefrologia. 2013; 33 (Suppl. 1): 1-28.

3. Obi Y, Mehrotra R, Rivara MB, Streja E, Rhee CM, Lau WL et al. Hidden hypercalcemia and mortality risk in incident hemodialysis patients. J Clin Endocrinol Metab. 2016; 101 (6): 2440-2449.

4. Björkman MP, Sorva AJ, Tilvis RS. Calculated serum calcium is an insufficient surrogate for measured ionized calcium. Arch Gerontol Geriatr. 2009; 49 (3): 348-350.

5. Obi Y, Nguyen DV, Streja E, Rivara MB, Rhee CM, Lau WL et al. Development and validation of a novel laboratory-specific correction equation for total serum calcium and its association with mortality among hemodialysis patients. J Bone Miner Res. 2017; 32 (3): 549-559.

6. limori S, Mori Y, Akita W, Kuyama T, Takada S, Asai T et al. Diagnostic usefulness of bone mineral density and biochemical markers of bone turnover in predicting fracture in CKD stage 5D patients-a single-center cohort study. Nephrol Dial Transplant. 2012; 27 (1): 345-351.

7. West SL, Lok CE, Langsetmo L, Cheung AM, Szabo E, Pearce $D$ et al. Bone mineral density predicts fractures in chronic kidney disease. J Bone Miner Res. 2015; 30 (5): 913-919.

8. Yenchek RH, Ix JH, Shlipak MG, Bauer DC, Rianon NJ, Kritchevsky $\mathrm{SB}$ et al. Bone mineral density and fracture risk in older individuals with CKD. Clin J Am Soc Nephrol. 2012; 7 (7): 1130-1136.

9. Bouquegneau A, Salam S, Delanaye P, Eastell R, Khwaja A. Bone Disease after Kidney Transplantation. Clin J Am Soc Nephrol. 2016; 11 (7): 1282-1296.

10. Monier-Faugere MC, Mawad H, Qi Q, Friedler RM, Malluche $\mathrm{HH}$. High prevalence of low bone turnover and occurrence of osteomalacia after kidney transplantation. J Am Soc Nephrol. 2000; 11 (6): 1093-1099.

11. Iyer SP, Nikkel LE, Nishiyama KK, Dworakowski E, Cremers S, Zhang $\mathrm{C}$ et al. Kidney transplantation with early corticosteroid withdrawal: paradoxical effects at the central and peripheral skeleton. J Am Soc Nephrol. 2014; 25 (6): 1331-1341.

12. Akaberi $S$, Simonsen $O$, Lindergård $B$, Nyberg G. Can DXA predict fractures in renal transplant patients? Am J Transplant. 2008; 8 (12): 2647-2651.

13. Keronen S, Martola L, Finne P, Burton IS, Kröger H, Honkanen E. Changes in bone histomorphometry after kidney transplantation. Clin J Am Soc Nephrol. 2019; 14 (6): 894-903.

14. Ferreira GF, Montenegro FL de M, Machado DJ, lanhez LE, Nahas WC, David-Neto E. Parathyroidectomy after kidney transplantation: short-and long-term impact on renal function. Clinics. 2011; 66 (3): 431-435.

15. Schmid T, Müller P, Spelsberg F. Parathyroidectomy after renal transplantation: a retrospective analysis of long-term outcome. Nephrol Dial Transplant. 1997; 12 (11): 2393-2396.

16. Evenepoel P, Claes K, Kuypers D, Maes B, Bammens B, Vanrenterghem $Y$. Natural history of parathyroid function and calcium metabolism after kidney transplantation: A single-centre study. Nephrol Dial Transplant. 2004; 19 (5): 1281-1287.

17. Lou I, Foley D, Odorico SK, Leverson G, Schneider DF, Sippel RCH. How well does renal transplantation cure hyperparathyroidism? Ann Surg. 2015; 262 (4): 653-659.

18. Reinhardt W, Bartelworth $H$, Jockenhövel F, Schmidt-Gayk $H$, Witzke $\mathrm{O}$, Wagner $\mathrm{K}$ et al. Sequential changes of biochemical bone parameters after kidney transplantation. Nephrol Dial Transplant. 1998; 13 (2): 436-442.

19. Lobo PI, Cortez MS, Stevenson WPT. Normocalcemic hyperparathyroidism associated with relatively low 1:25 vitamin D levels post-renal transplant can be successfully treated with oral calcitriol. Clin Nephrol. 1995; 9 (4): 277.

20. Littbarski SA, Kaltenborn A, Gwiasda J, Beneke J, Arelin $V$, Schwager $Y$ et al. Timing of parathyroidectomy in kidney transplant candidates with secondary hyperparathryroidism: effect of pretransplant versus early or late post-transplant parathyroidectomy. Surgery. 2018; 163 (2): 373-380.

21. Callender GG, Malinowski J, Javid M, Zhang Y, Huang H, Quinn $\mathrm{CE}$ et al. Parathyroidectomy prior to kidney transplant decreases graft failure. Surgery. 2017; 161 (1): 44-50.

22. Apetrii M, Goldsmith D, Nistor I, Siriopol D, Voroneanu L, Scripcariu $D$ et al. Impact of surgical parathyroidectomy on 
chronic kidney disease-mineral and bone disorder (CKDMBD)-A systematic review and meta-analysis. PLoS One. 2017; 12 (11): 1-17.

23. FitzGerald RA, Sehgal AR, Nichols JA, McHenry CR. Factors predictive of emergency department visits and hospitalization following thyroidectomy and parathyroidectomy. Ann Surg Oncol. 2015; 22 Suppl 3: S707-S713.

24. Isaksson E, Almquist M, Seeberger A, Sterner G. Is low pretransplant parathyroid hormone a risk marker for cardiovascular disease in long-term follow-up of renal transplant recipients? Clin Exp Nephrol. 2018; 22 (5): 1188-1197.

25. Messa P, Regalia A, Alfieri CM, Cresseri D, Forzenigo L, Gandolfo MT et al. Current indications to parathyroidectomy in CKD patients before and after renal transplantation. J Nephrol. 2013; 26 (6): 1025-1032.

26. Moroni G, Tantardini F, Gallelli B, Quaglini S, Banfi G, Poli $F$ et al. The long-term prognosis of renal transplantation in patients with lupus nephritis. Am J Kidney Dis. 2005; 45 (5): 903-911.

27. Uhlig K, Berns JS, Kestenbaum B, Kumar R, Leonard MB, Martin $\mathrm{KJ}$ et al. KDOQI US Commentary on the 2009 KDIGO Clinical Practice Guideline for the Diagnosis, Evaluation, and Treatment of CKD-Mineral and Bone Disorder (CKD-MBD). Am J Kidney Dis. 2010; 55 (5): 773-799.

28. Kidney Disease: Improving Global Outcomes (KDIGO) CKD Work Group. KDIGO 2012 Clinical Practice Guideline for the Evaluation and Management of Chronic Kidney Disease. Kidney Int Suppl. 2013; 3 (1): 4-4.

29. Zhang L, Liu X, Li H. Long-term skeletal outcomes of primary hyperparathyroidism patients after treatment with parathyroidectomy: a systematic review and meta-analysis. Horm Metab Res. 2018; 50 (3): 242-249.

30. Punch JD, Thompson NW, Merion RM. Subtotal parathyroidectomy in dialysis-dependent and post-renal transplant patients. A 25-year single-center experience. Arch Surg. 1995; 130 (5): 538-542; discussion 542-543.

31. Tominaga $Y$, Uchida K, Haba T, Katayama A, Sato T, Hibi $Y$ et al. More than 1,000 cases of total parathyroidectomy with forearm autograft for renal hyperparathyroidism. Am J Kidney Dis. 2001; 38 (4 Suppl 1): S168-S171.

32. Sato T, Tominaga Y, Ueki T, Goto N, Matsuoka S, Katayama $A$ et al. Total parathyroidectomy reduces elevated circulating fibroblast growth factor 23 in advanced secondary hyperparathyroidism. Am J Kidney Dis. 2004; 44 (3): 481-487.

33. Dulfer RR, Franssen GJH, Hesselink DA, Hoorn EJ, van Eijck $\mathrm{CHJ}$, van Ginhoven TM. Systematic review of surgical and medical treatment for tertiary hyperparathyroidism. Br J Surg. 2017; 104 (7): 804-813.

34. Matstusoka S, Tominaga Y, Uno N, Goto N, Sato T, Katayama A et al. Calciphylaxis: a rare complication of patients who required parathyroidectomy for advanced renal hyperparathyroidism. World J Surg. 2005; 29 (5): 632-635.

35. Checheriță IA, Smarandache D, David C, Ciocâlteu A, Ion DA, Lascăr I. Vascular calcifications in chronic kidney disease--clinical management. Rom J Morphol Embryol. 2012; 53 (1): 7-13.

36. Nigwekar SU, Brunelli SM, Meade D, Wang W, Hymes J, Lacson E. Sodium thiosulfate therapy for calcific uremic arteriolopathy. Clin J Am Soc Nephrol. 2013; 8 (7): 1162-1170.

37. DeLoach SS, Joffe MM, Mai X, Goral S, Rosas SE. Aortic calcification predicts cardiovascular events and all-cause mortality in renal transplantation. Nephrol Dial Transplant. 2009; 24 (4): 1314-1319.

Correspondencia:

Dr. Juan Carlos Ramírez-Sandoval

E-mail: jc25wing@yahoo.com 